[RADIOCARbon, Vol. 18, No. 2, 1976, P. 161-171]

\title{
GLASGOW UNIVERSITY RADIOCARBON MEASUREMENTS VIII
}

M J STENHOUSE* and M S BAXTER

Department of Chemistry, University of Glasgow, Glasgow G12 8QQ

INTRODUCTION

The analytical facilities at Glasgow have been extended to include gas proportional $\left(\mathrm{CO}_{2}\right.$ and $\left.\mathrm{CH}_{4}\right)$ and liquid scintillation $\left(\mathrm{C}_{6} \mathrm{H}_{6}\right)$ counting laboratories. The results presented here were obtained during 1972-1974 using the $\mathrm{CO}_{2}$ gas counting system only. In brief, organic samples, after pretreatment as described in the text, are burned in a tube combustion unit and the evolved $\mathrm{CO}_{2}$ absorbed in $\mathrm{KOH}$ solution. $\mathrm{BaCO}_{3}$ is precipitated and acid-hydrolyzed in vacuo using $\mathrm{H}_{3} \mathrm{PO}_{4}$. Evolved $\mathrm{CO}_{2}$ is purified via adsorption/desorption on $\mathrm{CaO}$ and is stored prior to counting. The 2.6L proportional counter is surrounded by a gas-flow Geiger anticoincidence guard and $10 \mathrm{~cm}$ thick $\mathrm{Pb}$ shielding to reduce background count rates to ca $4.9 \mathrm{cpm}$ at $1 \mathrm{~atm}$ filling and barometric pressures. A barometric sensitivity in background of $-0.01 \mathrm{cpm} / \mathrm{mbar}$ is observed. Constant gas gain is ensured by monitoring the coincidence meson spectrum and normalizing the detector operating voltage. All sample activities are related to the NBS oxalic acid standard count rate which averages $14.71 \mathrm{cpm}$ at $1 \mathrm{~atm}$ filling pressure and $15^{\circ} \mathrm{C}$. Mass spectrometric assay of $\mathrm{CO}_{2}$ after counting is performed on a VG Micromass 602B instrument to a precision of $0.05 \% \circ( \pm 1 \sigma)$. Since uncertainties quoted on all results represent $1 \sigma$ counting errors alone, they are related to precision of measurement rather than accuracy. The bulk of data quoted here are connected with a long-term study of the medical aspects of artificial ${ }^{14} \mathrm{C}$ from nuclear weapon tests. These results should therefore be assessed in conjunction with those pub previously (Harkness and Walton, 1972; Farmer et al, 1972).

\section{ACKNOWLEDGMENTS}

This research was generously supported by a grant from the Medical Research Council. One of us (MJS) thanks the Natural Environment Research Council for financial aid. Our gratitude is extended to the many suppliers of samples. W A Harland, besides collecting tissue samples, added useful comment. Mass-spectrometric analyses owe much to A E Fallick while the technical assistance of A Miller is gratefully acknowledged.

\section{SAMPLE DESCRIPTIONS}

\section{ARCHAEOLOGIC SAMPLES}

While the principal objectives of the Glasgow labs lie in geochemical research, a small number of dating samples are assayed. Usually, measurements are made for intercalibration with other labs. All samples described here were pretreated by successive boiling in $5 \% \mathrm{NaOH} /$ distilled water/ $5 \% \mathrm{HCl}$ prior to combustion.

* Present address: Mount Soledad Radiocarbon Laboratory, University of California at San Diego, California 92093 


\section{GU-514. Kilphedir hut circles, Sutherland, Scotland

Charcoal (Birch-Betula). Comment: sample previously described and reported under GU-10, $1908 \pm 60$; GU-11, $2064 \pm 55$; GU-67, $1922 \pm$ 60; SRR-3, $2100 \pm 50$; L-1061, $2100 \pm 80$.

GU-515. Kilphedir hut circles, Sutherland, Scotland

$2045 \pm 65$

As GU-514.

95 BC

\section{GU-516. Wakefield, Yorkshire}

Wood (Quercus). Comment: sample previously described and reported under IGS-C14/65 (St-3399), $2585 \pm 100$ and SRR-7, $2569 \pm 80$.

\section{GU-517. Leeds, Yorkshire}

$4120 \pm 105$

Wood. Comment: sample previously described and reported under IGS-CI4/4 (St-3057), $4280 \pm 100$.

\section{GU-518. Leeds, Yorkshire}

$4155 \pm 70$

As GU-517, analyzed 1 yr later (1972).

GU-519. Leeds, Yorkshire 2205 BC

As GU-517, -518, analyzed 1973.

$4150 \pm 105$

2200 BC

\section{GU-520. Chesil Beach, Abbotsbury}

$3875 \pm 90$

Peat (Phragmites). Comment: sample previously described and reported under Q-1028, $4234 \pm 60$; Q-1029, $4251 \pm 60$; Q-1030, $5058 \pm 60$; $\mathrm{I}-5760,5770 \pm 100$; SRR-24, $4023 \pm 50$; SRR-25, $4095 \pm 60$. Wide range of reported dates due to intrusion of non-contemporaneous fractions of plant materials and to varying degrees of success in their removal.

\section{Ayios Epiktitos Vrysi, Cyprus series}

Samples from middle phase of Neolithic coastal settlement $9.7 \mathrm{~km}$ E of Kyrenia, Cyprus ( $35^{\circ} 20^{\prime}$ N, $33^{\circ} 26^{\prime}$ E). Samples coll by E J Peltenburg, Dept Extra-Mural Educ, Univ Glasgow.

\section{GU-521. Kyrenia 1}

$3105 \pm 130$

Wood charcoal from beside hearth in Passage A, Floor 5, $1.20 \mathrm{~m}$ below surface.

\section{GU-522. Kyrenia 2}

$5420 \pm 80$

Wood charcol around major hearth of below surface.

GU-523. Kyrenia 3

$5340 \pm 95$

Wood charcoal from same place as GU-522. 
GU-524. Kyrenia 4

\section{BC}

Wood charcoal flecks scattered in SW corner of Floor 2, House 1, $1.50 \mathrm{~m}$ below surface.

General Comment (EJP, MSB \& MJS): in all cases, the Kyrenia samples were of poor quality, finely dispersed charcoal grains heavily intermixed with soil. Laborious microscopic examinations and extractions were required to separate charcoal particles. Thus, even after pretreatment, the possibility of contamination remained significant. In addition, small size of samples necessitated $\mathrm{CO}_{2}$ dilution procedures for counting. GU-521, the least satisfactory sample, yielded an archaeologically unacceptable date as there is no 1 st to 2 nd millennium $\mathrm{BC}$ occupation on site. Remaining Kyrenia dates are slightly later than expected relative to results on similar sites (Birm-182, $5825 \pm 145$; Birm-337, $5740 \pm 140$ ).

Considered along with nuclear era intercalibration samples, GU-560562 and with weekly data on NBS oxalic acid standard samples, samples GU-514-524 imply satisfactorily accurate measurement.

\section{ATMOSPHERIC SAMPLES}

Troposheric ${ }^{14} \mathrm{C}$ activities over $\mathbf{U} \mathbf{K}$ have been monitored at Glasgow since 1968. Results presented here extend previously pub data and provide essential input information for dietary and human tissue studies in later sections. Also, they record ${ }^{14} \mathrm{C}$ fluctuations induced by continued nuclear weapon testing. Monthly tropospheric $\mathrm{CO}_{2}$ samples from colln stas at Lerwick $\left(60^{\circ} 08^{\prime} \mathrm{N}, 01^{\circ} 11^{\prime} \mathrm{W}\right)$, Snowdon $\left(53^{\circ} 03^{\prime} \mathrm{N}, 04^{\circ} 00^{\prime} \mathrm{W}\right)$, and Harwell $\left(51^{\circ} 31^{\prime} \mathrm{N}, 01^{\circ} 20^{\prime} \mathrm{W}\right)$ were obtained by exposure of carbonate-free $4 \mathrm{M}$ KOH solution to the atmosphere for each calendar month. After precipitation as $\mathrm{BaCO}_{3}$, samples were hydrolyzed with $\mathrm{H}_{3} \mathrm{PO}_{4}$. While analysis of every sample was not possible, representative samples were measured.

\section{Lerwick series, 1971-1973}

Samples coll by Meteorol Office in ventilated E hut, Lerwick.

\begin{tabular}{crccc}
\hline $\begin{array}{c}\text { Sample } \\
\text { no. }\end{array}$ & Date coll & $\delta^{14} \mathrm{C} \%$ & $\delta^{13} \mathrm{C} \%{ }^{*}$ & $\Delta \%$ \\
\hline GU-525 & July 1971 & $53.3 \pm 1.2$ & -18.3 & $51.2 \pm 1.2$ \\
GU-526 & Sept 1971 & $54.4 \pm 1.1$ & -19.4 & $52.7 \pm 1.2$ \\
GU-527 & April/May 1972 & $49.3 \pm 0.8$ & $(-16.2)$ & $46.7 \pm 0.8$ \\
GU-528 & July 1972 & $51.8 \pm 0.8$ & -14.8 & $48.7 \pm 0.8$ \\
GU-529 & Oct/Nov 1972 & $46.7 \pm 0.8$ & -16.0 & $44.1 \pm 0.8$ \\
GU-530 & Feb 1973 & $44.8 \pm 0.8$ & -16.1 & $42.2 \pm 0.8$ \\
GU-531 & May 1973 & $45.7 \pm 1.0$ & $(-16.2)$ & $43.1 \pm 1.0$ \\
\hline
\end{tabular}

* Where $\delta^{13} \mathrm{C}$ measurement was not possible, normally as a result of dilution of sample $\mathrm{CO}_{2}$, a mean $\delta^{13} \mathrm{C}$ for the sample type is assumed and recorded in parenthesis. For these samples a $\pm 1 \sigma$ error of $1 \%$ is estimated, $c f 0.05 \%$ for directly measured samples. 


\section{Snowdon series, 1971-1973}

Samples coll by Central Electricity Generating $\mathrm{Bd}$ in ventilated cabinet at Cwm Dyli Power Sta on E slope of Mt Snowdon.

\begin{tabular}{lrccc}
\hline $\begin{array}{c}\text { Sample } \\
\text { no. }\end{array}$ & Date coll & $\delta^{14} \mathrm{C} \%$ & $\delta^{13} \mathrm{C} \%$ o & $\Delta \%$ \\
\hline GU-532 & April 1971 & $52.0 \pm 0.9$ & -17.4 & $49.7 \pm 0.9$ \\
GU-533 & July 1971 & $55.8 \pm 1.2$ & -14.2 & $52.4 \pm 1.2$ \\
GU-534 & Sept 1971 & $51.6 \pm 0.9$ & $(-16.0)$ & $48.9 \pm 0.9$ \\
GU-535 & Dec 1972 & $44.9 \pm 0.8$ & -15.8 & $42.3 \pm 0.8$ \\
GU-536 & March 1973 & $45.5 \pm 0.9$ & $(-16.0)$ & $42.8 \pm 0.9$ \\
GU-537 & June 1973 & $47.1 \pm 0.9$ & $(-16.0)$ & $44.5 \pm 0.9$ \\
\hline
\end{tabular}

Harwell series, 1971-1973

Samples coll by U K Atomic Energy Comm at site adjacent to A E R E, Harwell.

\begin{tabular}{lrccc}
\hline $\begin{array}{c}\text { Sample } \\
\text { no. }\end{array}$ & Date coll & $\delta^{14} \mathrm{C} \%$ & $\delta^{13} \mathrm{C} \%$ o & $\Delta \%$ \\
\hline GU-538 & March 1971 & $47.2 \pm 1.0$ & -20.8 & $45.9 \pm 1.0$ \\
GU-539 & June 1971 & $63.3 \pm 1.0$ & -18.7 & $61.3 \pm 1.0$ \\
GU-540 & Sept 1971 & $58.4 \pm 0.8$ & -19.8 & $56.8 \pm 0.8$ \\
GU-541 & Aug/Sept 1972 & $57.8 \pm 0.9$ & -20.4 & $56.3 \pm 0.9$ \\
GU-542 & Jan 1973 & $46.9 \pm 0.8$ & -21.8 & $45.9 \pm 0.8$ \\
GU-543 & May 1973 & $46.1 \pm 0.8$ & $(-20.7)$ & $44.8 \pm 0.9$ \\
\hline
\end{tabular}

General Comment: ${ }^{14} \mathrm{C}$ variations, both temporal and spatial, continue in $\mathrm{U} \mathrm{K}$ tropospheric air as the major artificial input of the early 1960's equilibrates with the oceanic and biospheric reservoirs and as nuclear testing, on a smaller scale, persists. Fluctuations are superimposed on a decreasing trend averaged at ca $2.5 \% / \mathrm{yr}$ by 1972 . Aided by the Suess effect, atmospheric ${ }^{14} \mathrm{C}$ activities should decrease below the natural level by $\mathrm{AD} 2000$. Results of the Harwell series indicate no major ${ }^{14} \mathrm{C}$ release from the adjacent nuclear reactor. Taken together, the atmospheric data provide a basis for evaluation of dietary and tissue results of the following sections.

\section{DIETARY SAMPLES}

A realistic assessment of ${ }^{14} \mathrm{C}$ levels and variations in human tissues requires accurate information on the ${ }^{14} \mathrm{C}$ input via diet. Systematic investigation of ${ }^{14} \mathrm{C}$ activities of all constituents of diet is, however, impracticable. In this study, samples of uncertain relationship to atmospheric ${ }^{14} \mathrm{C}$ concentrations, $i e$, fish and meat tissues, were analyzed. Meat samples were pretreated identically to human tissues while fish were freeze-dried after removal of bones. All samples quoted here were purchased in Glasgow stores. Included in this section are results of intercalibration samples of bovine tissues supplied by $\mathrm{R} M$ Chatters, Washington State Univ. 
Fish samples, series

Samples obtained fresh, March 1973, except GU-550, which was canned.

\begin{tabular}{llccc}
\hline $\begin{array}{c}\text { Sample } \\
\text { no. }\end{array}$ & \multicolumn{1}{c}{ Species } & $\delta^{14} \mathrm{C}_{\%}$ & $\delta^{13} \mathrm{C} \%$ o & $\Delta \%$ \\
\hline GU-544 & Herring & $14.1 \pm 0.8$ & -19.7 & $12.9 \pm 0.8$ \\
GU-545 & Whiting & $17.6 \pm 0.8$ & -17.3 & $15.8 \pm 0.8$ \\
GU-546 & Herring (smoked) & $14.3 \pm 0.9$ & -19.4 & $13.0 \pm 0.9$ \\
GU-547 & Cod & $14.6 \pm 1.0$ & -17.8 & $12.9 \pm 1.0$ \\
GU-548 & Plaice & $29.0 \pm 0.8$ & -15.3 & $26.5 \pm 0.8$ \\
GU-549 & Haddock & $18.2 \pm 0.8$ & -18.3 & $16.6 \pm 0.8$ \\
GU-550 & Salmon & $14.9 \pm 0.7$ & -22.1 & $14.2 \pm 0.7$ \\
\hline
\end{tabular}

\section{Meat samples, series}

Samples obtained fresh, Jan 1972. For each sample, whole tissue (W), protein $(\mathrm{P})$ and lipid $(\mathrm{L})$ fractions were analyzed.

\begin{tabular}{llccc}
\hline $\begin{array}{l}\text { Sample } \\
\text { no. }\end{array}$ & Description & $\delta^{14} \mathrm{C} \%$ & $\delta^{13} \mathrm{C} \%$ o & $\Delta \%$ \\
\hline GU-551 & Lamb's heart (W) & $44.4 \pm 1.0$ & -28.3 & $45.3 \pm 1.0$ \\
GU-552 & Lamb's heart (L) & $49.4 \pm 1.1$ & -31.1 & $51.2 \pm 1.1$ \\
GU-553 & Lamb's heart (P) & $46.4 \pm 1.1$ & -25.5 & $46.6 \pm 1.1$ \\
GU-554 & Lamb's kidney (W) & $54.5 \pm 2.4$ & -29.9 & $56.0 \pm 2.5$ \\
GU-555 & Lamb's kidney (L) & $50.7 \pm 1.2$ & -25.9 & $51.0 \pm 1.2$ \\
GU-556 & Lamb's kidney (P) & $59.5 \pm 1.3$ & -25.0 & $59.5 \pm 1.3$ \\
GU-557 & Cow's liver (W) & $43.8 \pm 1.0$ & -26.5 & $44.2 \pm 1.0$ \\
GU-558 & Cow's liver (L) & $60.0 \pm 1.0$ & -28.7 & $61.2 \pm 1.0$ \\
GU-559 & Cow's liver (P) & $47.9 \pm 1.1$ & -24.8 & $47.6 \pm 1.1$ \\
\hline
\end{tabular}

\section{Animal tissues, series}

Samples subm by R M Chatters, Washington State Univ. All samples from animals slaughtered 1971. Data are presented as $\Delta(\%)$ with corresponding WSU sample nos. and results that are based on an assumed $\delta^{13} \mathrm{C}$ of $-25 \%$.

\begin{tabular}{lllr}
\hline Sample no. & Description & $\Delta_{\%}^{\circ}(\mathrm{GU})$ & $\Delta \%$ (WSU) \\
\hline GU-560/WSU-1189 & Cow heart & $51.5 \pm 1.1$ & $48.2 \pm 1.7$ \\
GU-561/WSU-1187 & Cow cartilage & $31.5 \pm 0.9$ & $31.8 \pm 1.9$ \\
GU-562/WSU-1168 & Cow muscle & $58.3 \pm 1.0$ & $60.7 \pm 2.8$ \\
\hline
\end{tabular}

General Comment: the data indicate that ${ }^{14} \mathrm{C}$ contents of dietary samples analyzed reflect, fairly closely, environments of their origin, ie, surface ocean and troposphere/plant biosphere, respectively. The low value of bovine cartilage, GU-561, reflects the slow turnover of that material. 
Intercalibration samples show excellent agreement. Since the object of the program was to evaluate ${ }^{14} \mathrm{C}$ content of diet for any given time during the nuclear era, the above experimental data were accompanied by a theoretical survey of dietary habits and statistics (Stenhouse, 1974). Account was taken of ${ }^{14} \mathrm{C}$ lag-time effects in food production, of importation particularly from $\mathrm{S}$ hemisphere, and of protein, fat, and carbohydrate contents of foodstuffs. From this study, the ${ }^{14} \mathrm{C}$ content of $\mathrm{U} K$ diet over any desired time period ' $\mathrm{i}$ ' can be calculated via the following equation,

$$
\begin{gathered}
\Delta_{\mathrm{D}}{ }^{\mathrm{ni}}=0.24 \Delta_{\mathrm{TN}}{ }^{\mathrm{ni}}+0.19 \Delta_{\mathrm{TN}}{ }^{(\mathrm{n}-1) \mathrm{i}}+0.16 \Delta_{\mathrm{TN}}{ }^{(\mathrm{n}-2) \mathrm{i}}+0.28 \Delta_{\mathrm{TN}}{ }^{\mathrm{J}}+0.02 \Delta_{\mathrm{TS}}{ }^{\mathrm{ni}} \\
+0.035 \Delta_{\mathrm{TS}}{ }^{(\mathrm{n}-2) \mathrm{i}}+0.035 \Delta_{\mathrm{TS}}{ }^{\mathrm{J}}+0.03 \Delta_{\mathrm{M}^{\mathrm{ni}}}+0.01 \Delta_{\mathrm{M}}{ }^{(\mathrm{n}-1) \mathrm{i}}
\end{gathered}
$$

where subscripts TN, TS, and $M$ represent $N$ troposphere, $S$ troposphere and surface ocean, respectively, and superscript $\mathrm{J}$ refers to the preceding period of maximum photosynthesis. In practice, ' $\mathrm{i}$ ' was taken as 6 mos. The equation enables definition of $\mathrm{U} \mathrm{K}$ dietary ${ }^{14} \mathrm{C}$ intake at any time after nuclear testing commenced. Full details of the derivation of this and related dietary models will be pub elsewhere (Stenhouse and Baxter, mss in preparation). Its significance here is that it enables quantitative treatment of human tissue ${ }^{14} \mathrm{C}$ variations.

\section{HUMAN TISSUE SAMPLES}

Observation of the rate at which human tissues responded to the pulse of artificial ${ }^{14} \mathrm{C}$ from nuclear weapon testing should enable assessment of the kinetics of tissue carbon turnover. Also, estimated present and future radiation dose levels to body tissues are possible. The following data add to those already obtained at this lab (Harkness and Walton, 1972; Farmer et al, 1972). Samples were coll at post-mortem examinations no later than 5 days after death. For each specimen, age at death, sex, and cause of death were recorded. All tissues, except where stated, were of Glasgow origin. Samples were first washed with distilled water to remove external traces of blood and were dissected free from extraneous fat, homogenized with 10 to $15 \mathrm{ml}$ distilled water to purée texture, and freeze-dried overnight; 2 to $5 \mathrm{~g}$ dried material was obtained for combustion: In several cases, analyses were performed on whole tissue $(\mathrm{W})$, protein $(\mathrm{P})$ and lipid (L) fractions of each sample. Protein- and lipid-rich components were separated, using Bloor's solvent (ethanol/ diethyl ether, $3: 1 \mathrm{v} / \mathrm{v}$ ). Solvent contamination was shown to be negligible by using ${ }^{14} \mathrm{C}$-labeled solvents for several extractions at activities sufficient to produce $10 \mathrm{cpm} /$ counter fill at $1 \%$ contamination level. Aortic samples from victims of atherosclerosis were pretreated for lipid extraction using a chloroform/methanol mixture $(2: 1 \mathrm{v} / \mathrm{v})$ followed by dissolution in diethyl ether/hexane $(1: 1 \mathrm{v} / \mathrm{v})$ and washing with saline solution to remove non-lipid contaminants. The samples analyzed therefore represent the combined cholesterol, cholesterol esters, free fatty acids and phospholipids of arterial deposits. 


\section{9-year-old human tissues, series}

Samples from a 39-yr-old male who died Aug 1971 of coronary artery disease.

\begin{tabular}{llccc}
\hline $\begin{array}{c}\text { Sample } \\
\text { no. }\end{array}$ & Tissue & $\delta^{14} \mathrm{C} \%$ & $\delta^{13} \mathrm{C} \%$ & $\Delta \%$ \\
\hline GU-563 & Liver & $43.0 \pm 1.2$ & $(-22.5)$ & $42.3 \pm 1.2$ \\
GU-564 & Brain & $49.3 \pm 0.9$ & -21.1 & $48.1 \pm 0.9$ \\
GU-565 & Spleen & $39.5 \pm 1.0$ & $(-21.7)$ & $38.6 \pm 1.0$ \\
GU-566 & Thyroid & $38.6 \pm 1.5$ & $(-22.5)$ & $37.9 \pm 1.5$ \\
GU-567 & Pancreas & $46.1 \pm 1.2$ & $(-24.7)$ & $46.0 \pm 1.2$ \\
GU-568 & Heart & $40.8 \pm 1.2$ & -21.2 & $39.8 \pm 1.2$ \\
\hline
\end{tabular}

\section{2-yr-old human tissues, series}

Samples from a 42-yr-old male who died Sept 1971 of rheumatic heart disease.

\begin{tabular}{llccc}
\hline $\begin{array}{c}\text { Sample } \\
\text { no. }\end{array}$ & Tissue & $\delta^{14} \mathrm{C}^{\circ}$ & $\delta^{13} \mathrm{C} \%$ o & $\Delta \%$ \\
\hline GU-569 & Heart & $52.3 \pm 1.5$ & $(-21.6)$ & $51.3 \pm 1.5$ \\
GU-570 & Kidney & $34.5 \pm 0.9$ & $(-22.6)$ & $33.9 \pm 0.9$ \\
GU-571 & Liver & $48.5 \pm 0.9$ & $(-22.5)$ & $47.7 \pm 1.0$ \\
GU-572 & Brain & $52.7 \pm 1.0$ & $(-22.4)$ & $51.9 \pm 1.0$ \\
GU-573 & Lung & $38.5 \pm 1.0$ & $(-22.5)$ & $37.8 \pm 1.0$ \\
GU-574 & Thyroid & $44.5 \pm 0.9$ & -21.1 & $43.3 \pm 0.9$ \\
GU-575 & Spleen & $44.4 \pm 2.5$ & $(-21.7)$ & $43.5 \pm 2.5$ \\
\end{tabular}

\section{1-yr-old human tissues, series} accident.

Samples from a 21-yr-old male who died Sept 1971 in road traffic

\begin{tabular}{llccc}
\hline $\begin{array}{c}\text { Sample } \\
\text { no. }\end{array}$ & Tissue & $\delta^{14} \mathrm{C} \%$ & $\delta^{13} \mathrm{C} \%$ & $\Delta \%$ \\
\hline GU-576 & Heart & $43.3 \pm 1.4$ & $(-21.6)$ & $42.4 \pm 1.4$ \\
GU-577 & Spleen & $46.9 \pm 0.8$ & -22.1 & $46.0 \pm 0.8$ \\
GU-578 & Liver & $55.1 \pm 0.9$ & -23.3 & $54.6 \pm 0.9$ \\
GU-579 & Thyroid & $44.2 \pm 1.5$ & $(-22.1)$ & $43.3 \pm 1.6$ \\
GU-580 & Kidney & $52.0 \pm 1.6$ & $(-22.6)$ & $51.2 \pm 1.6$ \\
GU-581 & Testes & $56.4 \pm 1.3$ & $(-23.7)$ & $56.0 \pm 1.3$ \\
GU-582 & Lung & $54.7 \pm 1.6$ & $(-22.5)$ & $53.9 \pm 1.6$ \\
GU-583 & Muscle & $56.8 \pm 1.3$ & -22.0 & $55.9 \pm 1.3$ \\
GU-584 & Pancreas & $52.3 \pm 1.1$ & -24.7 & $52.2 \pm 1.1$ \\
& & & &
\end{tabular}




\section{1-yr-old human tissues, series}

Samples from a 21-yr-old female who died Sept 1971 of acute diabetes.

\begin{tabular}{clccc}
\hline $\begin{array}{c}\text { Sample } \\
\text { no. }\end{array}$ & Tissue & $\delta^{14} \mathrm{C}_{\%}$ & $\delta^{13} \mathrm{C} \%$ & $\Delta \%$ \\
\hline GU-585 & Kidney & $53.0 \pm 1.2$ & -25.3 & $53.1 \pm 1.2$ \\
GU-586 & Muscle & $52.4 \pm 1.3$ & $(-22.5)$ & $51.7 \pm 1.3$ \\
GU-587 & Spleen & $55.2 \pm 1.6$ & -23.5 & $54.8 \pm 1.6$ \\
GU-588 & Heart & $55.6 \pm 1.3$ & -21.4 & $54.5 \pm 1.3$ \\
GU-589 & Liver & $55.5 \pm 1.0$ & -24.0 & $55.2 \pm 1.0$ \\
GU-590 & Lung & $51.9 \pm 1.1$ & -22.5 & $51.1 \pm 1.1$ \\
GU-591 & Pancreas & $55.2 \pm 1.1$ & -23.3 & $54.6 \pm 1.1$ \\
GU-592 & Thyroid & $51.6 \pm 1.1$ & -22.1 & $50.7 \pm 1.1$ \\
GU-593 & Brain & $53.1 \pm 1.1$ & -22.4 & $52.3 \pm 1.1$ \\
GU-594 & Ovaries & $51.3 \pm 2.0$ & $(-22.5)$ & $50.6 \pm 2.0$ \\
\hline
\end{tabular}

\section{5-yr-old human tissues, series} dent.

Samples from a 5-yr-old male who died Oct 1971 in road traffic acci-

\begin{tabular}{llccc}
\hline $\begin{array}{c}\text { Sample } \\
\text { no. }\end{array}$ & Tissue & $\delta^{14} \mathrm{C} \%$ & $\delta^{13} \mathrm{C} \%$ & $\Delta \%$ \\
\hline GU-595 & Muscle & $53.5 \pm 1.0$ & -21.7 & $52.5 \pm 1.0$ \\
GU-596 & Kidney & $51.2 \pm 1.4$ & $(-22.6)$ & $50.4 \pm 1.5$ \\
GU-597 & Spleen & $45.8 \pm 0.8$ & $(-21.7)$ & $44.9 \pm 0.8$ \\
GU-598 & Brain & $51.7 \pm 0.9$ & -21.1 & $50.5 \pm 0.9$ \\
GU-599 & Pancreas & $53.6 \pm 1.1$ & -24.7 & $53.5 \pm 1.2$ \\
GU-600 & Liver & $53.4 \pm 0.9$ & -22.8 & $52.8 \pm 0.9$ \\
\hline
\end{tabular}

\section{7-yr-old human tissues, series}

Samples from a 37 -yr-old male who died April 1972 of cardiac failure.

\begin{tabular}{clccc}
$\begin{array}{c}\text { Sample } \\
\text { no. }\end{array}$ & Tissue & $\delta^{14} \mathrm{C} \%$ & $\delta^{13} \mathrm{C} \%$ & $\Delta \%$ \\
\hline GU-601 & Heart & $48.7 \pm 1.0$ & -20.4 & $47.3 \pm 1.0$ \\
GU-602 & Liver & $49.1 \pm 0.9$ & -22.5 & $48.4 \pm 0.9$ \\
GU-603 & Testes & $50.2 \pm 1.8$ & -23.5 & $49.8 \pm 1.8$ \\
\hline
\end{tabular}




\section{7-yr-old human tissues, series}

Samples from a 67-yr-old male who died Jan 1973 of cardiac failure.

\begin{tabular}{llccc}
\hline $\begin{array}{l}\text { Sample } \\
\text { no. }\end{array}$ & Tissue & $\delta^{14} \mathrm{C} \%$ & $\delta^{13} \mathrm{C} \%$ & $\Delta \%$ \\
\hline GU-604 & Kidney (W) & $47.5 \pm 0.8$ & -21.8 & $46.6 \pm 0.8$ \\
GU-605 $\dagger$ & Kidney (P) & $49.8 \pm 0.9$ & -20.5 & $48.5 \pm 0.9$ \\
GU-606 & Heart (W) & $47.7 \pm 0.8$ & -21.9 & $46.8 \pm 0.8$ \\
GU-607 $\dagger$ & Heart (L) & $51.2 \pm 0.8$ & -24.6 & $51.1 \pm 0.8$ \\
GU-608 $\dagger$ & Heart (P) & $46.4 \pm 0.8$ & -21.8 & $45.4 \pm 0.8$ \\
GU-609 & Liver (W) & $50.2 \pm 0.8$ & -22.4 & $49.4 \pm 0.8$ \\
GU-610 & Liver (P) & $50.2 \pm 0.9$ & $(-22.5)$ & $49.4 \pm 1.0$ \\
GU-611 & Liver (L) & $49.4 \pm 1.2$ & $(-22.5)$ & $48.7 \pm 1.2$ \\
\hline
\end{tabular}

$\dagger$ Sample extracted using ${ }^{14} \mathrm{C}$-labeled solvents.

\section{7-yr-old human tissues, series}

Samples from an 87-yr-old female who died Jan 1973 of cardiac failure.

\begin{tabular}{llccc}
\hline $\begin{array}{c}\text { Sample } \\
\text { no. }\end{array}$ & Tissue & $\delta^{14} \mathrm{C} \%$ & $\delta^{13} \mathrm{C} \%$ & $\Delta \%$ \\
\hline GU-612 & Liver (W) & $48.8 \pm 0.9$ & -22.4 & $48.0 \pm 0.9$ \\
GU-613 & Liver (P) & $50.4 \pm 1.0$ & -20.7 & $49.1 \pm 1.0$ \\
GU-614 & Kidney (W) & $47.3 \pm 0.9$ & -21.3 & $46.2 \pm 0.9$ \\
GU-615 & Kidney (P) & $49.2 \pm 0.8$ & -20.6 & $47.9 \pm 0.8$ \\
GU-616 & Spleen (P) & $52.4 \pm 0.9$ & -20.8 & $51.1 \pm 0.9$ \\
\hline
\end{tabular}

\section{4-yr-old human tissues, series}

Samples from a 64-yr-old male who died Nov 1972 of cardiac failure.

\begin{tabular}{clccc}
$\begin{array}{c}\text { Sample } \\
\text { no. }\end{array}$ & Tissue & $\delta^{14} \mathrm{C} \%$ & $\delta^{13} \mathrm{C} \%$ o & $\Delta \%$ \\
\hline GU-617 & Kidney (W) & $47.6 \pm 0.9$ & -20.8 & $46.3 \pm 0.9$ \\
GU-618 & Kidney (P) & $49.4 \pm 1.0$ & -21.5 & $48.4 \pm 1.0$ \\
GU-619 & Liver (W) & $48.6 \pm 0.8$ & -21.3 & $47.5 \pm 0.8$ \\
GU-620 & Liver (P) & $49.9 \pm 0.9$ & -20.9 & $48.7 \pm 0.9$ \\
GU-621 & Liver (L) & $47.2 \pm 1.1$ & -25.0 & $47.2 \pm 1.1$ \\
GU-622 & Spleen (W) & $44.1 \pm 0.9$ & $(-21.7)$ & $43.2 \pm 1.0$ \\
GU-623 & Spleen (P) & $44.0 \pm 0.9$ & -20.5 & $42.8 \pm 0.9$ \\
GU-624 & Lung (W) & $44.8 \pm 1.2$ & -22.2 & $44.0 \pm 1.2$ \\
GU-625 & Lung (P) & $47.3 \pm 2.2$ & $(-22.5)$ & $46.5 \pm 2.2$ \\
GU-626 & Heart (P) & $42.2 \pm 0.9$ & -20.4 & $40.9 \pm 0.9$ \\
\hline
\end{tabular}




\section{1-yr-old human tissues, series}

Samples from a 61-yr-old male who died Oct 1972 of carcenoma of the lung.

\begin{tabular}{clccc}
\hline $\begin{array}{c}\text { Sample } \\
\text { no. }\end{array}$ & Tissue & $\delta^{14} \mathrm{C} \%$ & $\delta^{13} \mathrm{C} \%$ co & $\Delta \%$ \\
\hline GU-627 & Heart (W) & $51.1 \pm 0.8$ & -22.8 & $50.4 \pm 0.8$ \\
GU-628 & Heart (P) & $46.0 \pm 1.0$ & -21.1 & $44.9 \pm 1.0$ \\
GU-629 & Kidney (W) & $46.5 \pm 0.8$ & -20.8 & $45.3 \pm 0.8$ \\
GU-630 & Kidney (P) & $44.4 \pm 1.0$ & $(-22.6)$ & $43.7 \pm 1.0$ \\
GU-631 & Spleen (W) & $44.3 \pm 0.9$ & -22.0 & $43.4 \pm 0.9$ \\
GU-632 & Spleen (P) & $47.5 \pm 1.0$ & -20.8 & $46.2 \pm 1.0$ \\
GU-633 & Liver (W) & $49.2 \pm 0.9$ & $(-22.5)$ & $48.5 \pm 1.0$ \\
GU-634 & Liver (P) & $45.8 \pm 1.1$ & -22.3 & $45.0 \pm 1.1$ \\
\hline
\end{tabular}

\section{Tumor tissues, series}

Samples from individuals who died of cancer of the particular diseased tissue analyzed. Data from undiseased material are included for comparison.

\begin{tabular}{ccccc}
\hline $\begin{array}{c}\text { Sample } \\
\text { no. }\end{array}$ & Description & $\delta^{14} \mathrm{C} \%$ & $\delta^{13} \mathrm{C} \%$ & $\Delta \%$ \\
\hline GU-635 & $\begin{array}{c}\text { Liver (normal)-male, } \\
\text { 66 yr, died March 1973 } \\
\text { Liver (diseased)-donor } \\
\text { as GU-635 }\end{array}$ & $51.3 \pm 0.9$ & -20.8 & $50.0 \pm 0.9$ \\
GU-636 & $56.0 \pm 1.3$ & $(-22.5)$ & $55.2 \pm 1.4$ \\
GU-637 & $\begin{array}{c}\text { Spleen (normal)-male, } \\
\text { 63 yr, died Feb 1973 } \\
\text { Liver (diseased)-donor } \\
\text { as GU-637 }\end{array}$ & $52.0 \pm 0.8$ & $(-21.7)$ & $51.0 \pm 0.9$ \\
Gidney (diseased)-male & $53.8 \pm 1.2$ & $(-22.5)$ & $53.0 \pm 1.2$ \\
GU yr, died Feb 1973 & $49.4 \pm 0.8$ & $(-20.7)$ & $48.1 \pm 0.8$ \\
\hline
\end{tabular}

\section{Aortae lipids, series}

Samples from aortae of victims of atherosclerosis. Lipid samples GU640-642 from New Orleans, Louisiana. Samples GU-643 and -644 from Glasgow, represent intimal lipid and adventitial layer of the same artery.

\begin{tabular}{ccccc}
\hline $\begin{array}{c}\text { Sample } \\
\text { no. }\end{array}$ & Description & $\delta^{14} \mathrm{C} \%$ & $\delta^{13} \mathrm{C}_{\%}$ & $\Delta \%$ \\
\hline GU-640 & $\begin{array}{c}\text { Aorta (intimal lipid)- } \\
\text { male, 68 yr, died 1963 }\end{array}$ & $5.8 \pm 0.6$ & -20.2 & $4.8 \pm 0.6$ \\
GU-641 & $\begin{array}{c}\text { Aorta (intimal lipid)- } \\
\text { male, 67 yr, died 1964 }\end{array}$ & $10.7 \pm 0.9$ & $(-20.3)$ & $9.7 \pm 0.9$ \\
\hline
\end{tabular}




\begin{tabular}{ccccc}
\hline $\begin{array}{c}\text { Sample } \\
\text { no. }\end{array}$ & Description & $\delta^{14} \mathrm{C} \%$ & $\delta^{13} \mathrm{C} \%$ & $\Delta \%$ \\
\hline GU-642 & $\begin{array}{c}\text { Aorta (intimal lipid)- } \\
\text { male, 69 yr, died 1964 }\end{array}$ & $18.9 \pm 0.8$ & -20.6 & $17.9 \pm 0.8$ \\
GU-643 & $\begin{array}{c}\text { Aorta (intimal lipid)- } \\
\text { female, 86 yr, died 1973 }\end{array}$ & $47.5 \pm 0.9$ & -24.0 & $47.1 \pm 0.9$ \\
GU-644 & $\begin{array}{c}\text { Aorta (adventitia)- } \\
\text { donor as GU-643 }\end{array}$ & $32.7 \pm 0.8$ & -21.1 & $31.6 \pm 0.8$ \\
\hline
\end{tabular}

General Comment: all human tissue samples reflect above natural ${ }^{14} \mathrm{C}$ levels due to nuclear testing. Human tissue ${ }^{14} \mathrm{C}$ activities scatter outside statistical errors of measurement as a result, we believe, of variations in rate of carbon turnover in different organs of the body. Box model calculations for both steady state and growing tissues coupled with dietary studies and observed ${ }^{14} \mathrm{C}$ levels, indicate variations of mean residence time for ${ }^{14} \mathrm{C}$ within the range $6 \pm 4$ yr for soft tissues (Stenhouse, 1974; Stenhouse and Baxter, mss in preparation). Application of bomb ${ }^{14} \mathrm{C}$ as a biochemical tracer is further demonstrated by ${ }^{14} \mathrm{C}$ activities of arterial lipid extracts that imply a lipid turnover in $>10 \mathrm{yr}$. This finding is significant for a fuller understanding of the timescale of development and treatment of atherosclerosis. Based on extensive data recently compiled on somatic and genetic risks from ionizing radiation, corresponding risk estimates for artificial ${ }^{14} \mathrm{C}$ have been prepared. These suggest that, as a result both of the Suess effect and of bomb ${ }^{14} \mathrm{C}$ equilibration, this source of radioactivity does not present a severe threat to mankind. Overall, the net extent of severe damage, over that from natural ${ }^{14} \mathrm{C}$ and in the absence of major nuclear tests in the future, is assessed at 225 offspring with genetic disorders, of which ca 10 will occur in 1st generation offspring, while 350 to 1000 persons will suffer ${ }^{14} \mathrm{C}$-induced leukemia. A similar range of persons affected by ${ }^{14} \mathrm{C}$-induced bone tumors is predicted. In a world population of $\sim 2.5 \times 10^{9}$ and over a period of several generations, such an incidence of damage will occur undetected.

REFERENCES

Farmer, J G, Stenhouse, M J, and Baxter, M S, 1972, Glasgow University radiocarbon measurements VI: Radiocarbon, v 14, p 326-330.

Harkness, D D and Walton, A, 1972, Glasgow University radiocarbon measurements IV: Radiocarbon, v 14, p 111-113.

Stenhouse, M J, 1974, Artificial production of ${ }^{14} \mathrm{C}$ from nuclear weapon tests and its uptake by man: PhD thesis, Univ Glasgow. 